\title{
Gobiernos abiertos, ciudadanía que decide. Transformación digital del mecanismo de presupuestos participativos en Bogotá
}

\author{
A.C García-Acevedo, M.Sc ${ }^{1}$, J.D Suarez Jurado, Soft Eng. ${ }^{1}$, O.E Roa-Quintero, Soft Eng. ${ }^{1}$ A. Torres- Ramírez, Spc. ${ }^{1}$ \\ and J.A García-Camargo, M.Sc ${ }^{1}$ \\ ${ }^{1}$ Universidad Nacional de Colombia, Colombia,acgarcia@unal.edu.co,jdsuarezj@unal.edu.co,oeroaq@unal.edu.co, \\ altorresra@unal.edu.co,jhagarciaca@unal.edu.co
}

Resumen-Los presupuestos participativos son una herramienta ideal para procesos democráticos de amplia convocatoria, pues permiten la integración de poblaciones marginalizadas de decisiones gubernamentales. Su carácter innovador ha trascendido por un lapso de 30 años. Este mecanismo surgió en Porto Alegre, Brasil, en 1989 y en la actualidad se implementa en más de 2500 municipalidades del mundo. El éxito reside en la versatilidad, el fortalecimiento de las relaciones ciudadanía-Estado, la inclusión de diversas poblaciones y la posibilidad de veedurías sobre el uso de los recursos públicos.

Los espacios digitales contemporáneos en el marco de la web 3.0 y 4.0 están orientados a promover experiencias, acceso a servicios, información, comunicación, creación, configuración de nuevas geografías y fortalecimiento de comunidades gracias a la virtualidad. Los espacios digitales ofrecen un panorama que potencializa los alcances de los presupuestos participativos, y la lógica de diálogo y democratización de decisiones en torno al dinero público.

La transformación digital ha encontrado un campo acelerado de desarrollo en la pandemia iniciada en 2020. En este contexto, y como resultado de múltiples factores, surge la oportunidad de crear la plataforma digital Bogotá Participa

con tres módulos: Causas Ciudadanas, Consultas Ciudadanas y Presupuestos Participativos, para Bogotá, Colombia.

Este artículo señala la experiencia de construcción de dicha plataforma desde perspectivas sociales, diseño gráfico y desarrollo de software, haciendo énfasis en el módulo de Presupuestos Participativos. Este documento expone los fundamentos, los retos y las potencialidades que una herramienta digital puede aportar al desarrollo de procesos democráticos.

Palabras clave-- Presupuestos Participativos, Gobierno Abierto, Transformación digital.

Abstract-Participatory Budgets are an ideal tool for widely held democratic processes, as they allow the integration of marginalized populations from government decisions. Its innovative character has transcended in a span of 30 years. It emerged in Porto Alegre, Brazil, in 1989 and is currently implemented in more than 2,500 municipalities around the world. Its success resides in versatility, the strengthening of citizenshipstate relations, the inclusion of diverse populations and the possibility of oversight over the use of public resources.

Digital Object Identifier (DOI):

http://dx.doi.org/10.18687/LEIRD2021.1.1.1

ISSN: 2414-6390 ISBN: 978-958-52071-9-6
Contemporary digital spaces within the framework of the web $3.0 v 4.0$ are aimed at promoting experiences, access to services, information, communication, creation, configuration of new geographies and strengthening of communities thanks to virtuality. Digital spaces offer a panorama that enhances the scope of participatory budgets and the logic of dialogue and democratization of decisions around public money.

The digital transformation has found an accelerated field of development in the pandemic that began in 2020. In this context, and as a result of multiple factors, the opportunity arises to create the digital platform Bogotá Participa with three clusters: Citizen Causes, Citizen Consultations and Participatory Budgets, for the city of Bogotá, Colombia.

This paper points out the experience of building the platform from social perspectives, graphic design and software development, with an emphasis on the participatory budgeting module. This document sets out the fundamentals, challenges and potential that a digital tool can contribute to the development of democratic processes.

Keywords-- Participatory Budgets, Open Government, Digital Transformation.

\section{INTRODUCCIÓN}

\section{A. Definición y contexto}

Los presupuestos participativos tienen su origen histórico en el proceso postdictadura militar de Brasil, a finales de la década de los ochenta. La razón principal de su concepción residió en la notable desconfianza de la población civil ante las formas de gobernanza y el uso excluyente de los recursos públicos por parte de las clases dirigentes de aquel entonces. Sumado a esto, el escenario postdictadura también profería un panorama de incertidumbre frente a las promesas y nuevas formas de democracia que se estaban formulando en el país. Los nuevos procesos democráticos de aquel entonces se centraron en la vinculación de poblaciones tradicionalmente marginalizadas y de barrios populares en la ciudad de Porto Alegre bajo el marco del Gobierno de Administración Popular [1]inspirado en los consejos populares implementados en Francia durante la década de los setenta y en la planificación estratégica del gobierno de Salvador Allende en Chile [2]. 
El modelo de presupuestos inicialmente funcionó bajo varios planes piloto y fue sobrepasando dificultades hasta que logró establecerse como un modelo replicable. Diversos escenarios de corrupción, desconfianza y marginalización de grupos poblacionales y falta de representación son problemáticas similares que enfrentan diversas comunidades alrededor del mundo. Es por esto que, para mediados de los noventa, su implementación se consolidó en varias ciudades de Brasil y Latinoamérica, y en las décadas posteriores fue acogido en el mundo en más de 2500 municipalidades (pequeños centros poblados y grandes capitales del mundo) como posibilidad de fortalecimiento de la democracia y la participación [3], [4]

\section{B. Potencialidades}

Numerosas investigaciones y evaluaciones hechas desde la academia y por entidades gubernamentales demuestran que, en los treinta años del surgimiento de la propuesta, los presupuestos participativos han mejorado en un nivel significativo la calidad de la democracia en los territorios donde se han implementado [3], [4]. Ejemplo de esto es la promoción de mejores prácticas de gobernabilidad y de espacios de empoderamiento para los ciudadanos. Existe evidencia de resultados positivos vinculados a usos específicos de los presupuestos participativos que incluye el aumento del gasto municipal en saneamiento y salud, el incremento del número de organizaciones de la sociedad civil y la disminución de las tasas de mortalidad infantil [5].

Los presupuestos participativos surgieron bajo el principio de que el dinero de los gobiernos es de carácter público y, por lo tanto, la población en general debe participar en procesos de decisión de los gastos prioritarios y en la formulación y ejecución de los proyectos dirigidos al bienestar de una comunidad. Bajo esta línea, y, en resumen, los presupuestos participativos nacen como una forma de reparar la deteriorada relación entre el Estado y la ciudadanía, crear formación política, fomentar corresponsabilidad entre gobiernos y ciudadanos, y facilitar formas de vigilancia y control sobre el destino de los dineros públicos. Además, buscan disuadir la corrupción mediante la transparencia administrativa y fiscal, fomentan espacios de formación política y de administración pública para los ciudadanos; procuran fomentar el diálogo y fortalecer las relaciones sociales al interior de las comunidades a través de asambleas y la elección de líderes sociales.

La versatilidad del modelo de presupuestos participativos permite su implementación en diferentes escalas poblacionales, organizaciones locales, pequeños poblados o grandes ciudades o regiones de un país [6],[7]Se adaptan a la envergadura económica de diversos territorios pues se pueden implementar territorios con presupuestos anuales limitados a
20 dólares por habitante, o en ciudades que tengan la posibilidad de invertir miles de dólares anuales en cada ciudadano [8]A su vez, según la voluntad política de los gobernantes, la formación de la ciudadanía y la capacidad de decisión de esta, será posible decidir sobre un pequeño porcentaje del presupuesto público hasta el total de los dineros destinados a un territorio y una necesidad específica [9].

Los presupuestos participativos van más allá de una consulta sobre la aplicación de los recursos económicos hacia una u otra obra pública pues abre la posibilidad de proponer las obras públicas y los montos que deben ser financiables. Por tanto, se puede sostener que el presupuesto participativo es un instrumento privilegiado por varias razones: al combinar el modelo democrático representativo con el participativo, constituye un híbrido equilibrado de participación política institucionalizada y participación ciudadana autónoma, y se entrelaza con formas de innovación y cocreación.

\section{Limitantes}

Frente a todas las potencialidades del modelo de presupuestos participativos y su implementación global también han surgido estudios que han permitido caracterizar sus limitantes y los retos a superar [3] para garantizar una implementación íntegra. Las dificultades se intersectan con las limitaciones que tienen los procesos de participación ciudadana en general: dificultades de naturaleza política y de carácter comunicativo. En cuanto a lo político, las barreras son de carácter estructural e institucional y son responsabilidad del Estado. Estas limitaciones se pueden listar de la siguiente forma: ausencia de imparcialidad y transparencia en procesos electorales, ejercicios de poder autoritarios, falta de rendición de cuentas, sesgos ideológicos en la impartición de justicia, extensos procesos burocráticos, ausencia de protocolos claros para el ejercicio de derechos, la carencia de instrumentos jurídicos de participación y los medios efectivos para ejecutarlos.

Las limitantes de carácter comunicativo empatan con las motivaciones que permitieron la creación de los presupuestos Participativos: la enorme desconfianza ciudadana hacia las instituciones y las leyes; no obstante, también se entrelaza con razones ajenas a la responsabilidad del Estado y recaen en la responsabilidad de los medios de comunicación y la difusión de información a distintas escalas y en distintas plataformas sociales y digitales [10]. Es posible caracterizar las dificultades y los retos a mejorar de la siguiente manera: i. carencia de información y comunicación entre representantes políticos y ciudadanos representados, exaltación de la individualidad, ii. el consumo y el poder financiero y la relegación de la condición política de los ciudadanos dentro de un estado de derecho democrático, iii. ausencia de solidaridad. Además de estos elementos, podrían sumarse dificultades relacionadas con los medios tradicionales de comunicación: 
comunicación unilateral, con propósito de recepción, sesgos en la información, complacencia editorial con sectores específicos de la sociedad $\mathrm{y}$, en consecuencia, la invisibilización de sectores específicos [5].

\section{Plataforma Bogotá Participa}

La creciente transformación digital ha permeado procesos culturales, políticos y económicos en las últimas décadas. Colombia se ha suscrito al acogimiento de estos procesos [11]y también a la implementación de los presupuestos participativos en gobiernos regionales y recientemente en la capital colombiana. Sus dos referentes principales son la ciudad de Pasto [11] y Caldas [12]. Bogotá, como capital del país y ciudad de más de ocho millones de habitantes, se ha caracterizado por la búsqueda de mecanismos de participación ciudadana y de herramientas alternativas que la incentiven. Es así cómo ha encontrado en los presupuestos participativos una herramienta que potencializa la participación democrática [10]En este contexto de transformación digital, surge la iniciativa de creación de la plataforma Bogotá Participa y sus tres módulos. En el primero de ellos, Presupuestos Participativos, la ciudadanía puede postular iniciativas para la transformación de sus territorios, mediante la posibilidad de asignar presupuesto, priorizar iniciativas y proponer soluciones a problemáticas que afecten a comunidades de cada una de las localidades de la ciudad de Bogotá. En el segundo módulo, titulado Consultas Ciudadanas, los residentes en Bogotá podrán consultar información relacionada con dinámicas sociales, fenómenos u opiniones relacionadas con grupos específicos de la población capitalina. En el tercer módulo, Causas Ciudadanas, los bogotanos podrán solicitar a las entidades gubernamentales la solución a problemáticas específicas que tienen incidencia en toda la ciudad o solamente en un territorio puntual, mediante el apoyo ciudadano a dichas propuestas.

En los siguientes apartados de este artículo se detalla la metodología en términos de diseño y desarrollo del software que permitió la creación de la plataforma Bogotá Participa para la ciudad de Bogotá, Colombia. Todo este trabajo fue realizado bajo el liderazgo de la Secretaría de Gobierno de la ciudad de Bogotá en 2020 y construido por el Laboratorio de Innovación, Creatividad y Nuevas Tecnologías de la Universidad Nacional de Colombia -LAB101-, basándose y complementando los propósitos y trabajos realizados por la Secretaría Distrital de Planeación de la ciudad y el Instituto Distrital de La Participación y Acción Comunal -IDPAC- en años anteriores[13]Este artículo finaliza con los resultados concretos: el desarrollo de los módulos mencionados de la plataforma y una guía "paso a paso" para la utilización abierta a la ciudadanía. Además, se concluye con una serie de recomendaciones orientadas a futuros procesos de similar propósito y envergadura.

\section{MetodoloGíA}

\section{A. Presupuestos participativos en contexto de Transformación digital}

La creciente transformación digital ha permeado procesos culturales, políticos y económicos en las últimas décadas [14]. Esta transformación digital fue impulsada aún más por el contexto de pandemia, que acogió a la virtualidad y a los desarrollos digitales como principales herramientas de comunicación, de toma de decisiones, de procesos laborales y académicos, entre otros. Es en este contexto que el propósito de la implementación del modelo de presupuestos participativos a nivel tecnológico potencializa su alcance [15].

El LAB101, Laboratorio de Innovación de la Facultad de Ingeniería de la Universidad Nacional de Colombia, es un espacio de trabajo que busca transformar de manera positiva la realidad de las organizaciones mediante la innovación y el uso de tecnologías [16]En ese sentido, la experiencia adquirida en diferentes iniciativas permite ver la tecnología como un medio y no como un fin. El LAB101 busca la forma cómo pueden aprovecharse diversas tecnologías para solucionar problemáticas o generar oportunidades de mejoramiento de la sociedad.

La primera ventaja que ofrecen los medios digitales y el aprovechamiento de las TIC es la participación ciudadana digital, en donde los procesos comunicativos son abiertos a un público general y la burocracia es minimizada [3]Además, los medios digitales permiten disminuir la centralización de la información: surgen medios alternativos para la comunicación, los ciudadanos nutren los flujos de información, y es posible crear estrategias para superar la asimetría de información entre distintos sectores de la sociedad. La superación de dicha asimetría rompe brechas entre gobernantes y gobernados y permite diálogos informados con resultados concretos. Los medios digitales, también permiten la inclusión de mayores personas en los procesos democráticos. Incluso aquellas que tradicionalmente se han manifestado poco interesadas en procesos sociales y políticos mediante campañas de difusión efectivas [15]

Una segunda ventaja se relaciona con los costos y gastos que involucra implementar modelos de presupuestos participativos en diferentes locaciones gracias a la interactividad multilateral [3]. Las tecnologías digitales permiten la interacción entre ciudadanos y los funcionarios del Estado y también la creación de plataformas de propuesta, deliberación, voto, transparencia de licitaciones y rendición de cuentas a través de foros en línea, chats y listas de correo y múltiples espacios novedosos que han surgido bajo la necesidad de trabajo colaborativo en los tiempos de pandemia [10]. En la intersección entre presupuesto participativo y 
medios digitales resulta más sencillo exigir a los gobernantes y a los representantes elegidos democráticamente el cumplimiento de sus obligaciones.

\section{B. Experiencia del Laboratorio}

Así, con este fundamento teórico, las perspectivas de transformación digital y uso de herramientas TIC, y la experiencia de creación de soluciones útiles a la medida, el LAB101 creó la plataforma Bogotá Participa para la Alcaldía Mayor de Bogotá. Este trabajo fue facilitado y articulado por la Secretaría de Gobierno Distrital. Los parámetros que siguió el LAB101, y el trabajo articulado, se suscriben a las perspectivas de Gobierno Abierto [17], que promueven la colaboración entre distintos sectores de la sociedad como eje fundamental de la gobernanza y toma de decisiones[18].

El trabajo articulado para la creación de la plataforma inició en junio de 2020 y finalizó en octubre del mismo año. Para llevarse a cabo, contó con la participación de un equipo multidisciplinar compuesto por diseñadores gráficos, una lingüista, desarrolladores de software, un publicista, una antropóloga y estudiantes de diversos perfiles.

Para lograr la articulación de los diferentes campos de experticia, las labores de cada uno de los profesionales involucrados en esta propuestas y las perspectivas de las dos entidades involucradas, el LAB101 decidió implementar el marco de trabajo SCRUM, que permitió la creación de etapas de trabajo quincenales, que facilitaron la supervisión de los procesos, evaluación de los grados de articulación entre las tareas pendientes y a su vez, permitió identificar puntos de cambio o necesidad de iteraciones para el desarrollo óptimo de la propuesta [19]. El marco de trabajo SCRUM resultó conveniente para minimizar los cercos de incertidumbre y las dificultades de operar en un tiempo corto de ejecución.

Desde cada enfoque profesional del LAB101 se abordaron distintos procedimientos y metodologías para dar solución a las necesidades de creación de la plataforma Bogotá Participa y los tres módulos que la componen. En términos generales, estos procedimientos consistieron en consultas bibliográficas, definición de referentes precisos, adaptación a necesidades particulares, exploración de ciudadanías, creación de propuestas específicas que fueron debatidas, realimentadas y ajustadas en diálogo con la Secretaría de Gobierno de la ciudad.

\section{Enfoque desde diseño}

El equipo de diseñadores gráficos del LAB101, además de ser los encargados de toda la apuesta estética, visual y comunicativa de la plataforma se concentraron en crear una herramienta de fácil acceso, que generará identidad dentro los ciudadanos y que, entre otras cosas, tuviera una comunicación verbal contundente, visual, asertiva y que dentro de las diferentes posibilidades de operación hubiese conexiones lógicas, intuitivas y de interacción llamativa. Para lograr un resultado ajustado a las expectativas de la Secretaría de Gobierno de Bogotá y, sobre todo, acorde y amigable para los ciudadanos, fue necesario hacer una consulta extensiva. Aquí cabe aclarar que Bogotá es un territorio culturalmente diverso, en términos de género, etnicidad, clase, entre otros factores que dificultan crear un producto apto para un proceso de participación democrático que tenga personalización pues en la búsqueda de la singularidad es posible caer la formulación de características que podrían resultar excluyentes para algunos grupos poblacionales. Por lo tanto, la perspectiva que se acogió para el diseño de la plataforma podría enmarcarse bajo los lineamientos conceptuales del Diseño Universal [20]. Un diseño capaz de generar experiencias particulares e identidad pero que su uso sea práctico y sencillo para un amplio espectro de la población.

\section{Benchmark}

El proceso de documentación y referenciación para la creación de la plataforma inició con la búsqueda de sitios web con similares características y propósitos. Para esto se hizo uso de la estrategia Benchmark, que identifica las mejores prácticas en cualquier tipo de organización que se haya ganado una reputación de excelencia en un área específica [21]. El Benchmark, en este caso, hizo una búsqueda de referentes web similares de presupuestos participativos bajo tres criterios de análisis: funcionalidad, diseño visual, diseño de interacción.

Uno de los referentes en términos de funcionalidad fue la página de presupuestos participativos de la ciudad de Madrid, España y de Barcelona. De allí se tomaron referentes para crear la disposición de la información introductoria de la página, la disposición de las cifras, ubicación geográfica de las propuestas y líneas de tiempo que mostrara el carácter progresivo del desarrollo del modelo de presupuestos participativos. También bajo el enfoque de funcionalidad, se tuvo en cuenta que la plataforma debería ser receptora de propuestas ciudadanas, el equipo del LAB101 tomó como referentes las propuestas de interacción de la página de peticiones del gobierno de Washington, EU, y páginas de peticiones de interés general como Change.org.

En términos de diseño visual, se consultaron diversas páginas que permiten la visualización de líneas de tiempo, el uso de fotografías e ilustraciones acordes a cada uno de los módulos. La propuesta final se decantó por el uso mayoritario de fotografías tomadas del banco de imágenes de la Alcaldía de Bogotá, siguiendo una línea gráfica en sintonía con la imagen oficial del distrito. 
Finalmente, en cuanto al diseño de interacción uno de las preocupaciones más relevantes por parte del LAB101 y la Secretaría fue la característica de Billetera Digital: dinero simbólico y limitado sobre todo para denotar el carácter decisorio por parte de los ciudadanos: a cada ciudadano inscrito en la plataforma se le asignó un presupuesto, una bolsa de dinero en pesos colombianos (simbólicos), para usar dentro del módulo de presupuestos participativos. El ciudadano tiene la posibilidad de ingresar a Bogotá Participa y seleccionar uno de los proyectos propuestos y manifestar su interés asignando una cantidad de dinero de esa bolsa personal y, de esta misma forma, apoyar otras propuestas. Todo esto permite flexibilidad y otorga un carácter formador y pedagógico a la decisión y desistimiento de los dineros asignados a los usuarios, que aunque son simbólicos homologan los dineros reales sobre los que cada ciudadano tiene derecho a decidir.

Para la característica de Billetera Digital, se tuvieron en cuenta aplicaciones bancarias como Nequi, y portales que permiten transacciones monetarias y ofrecen un historial de compras y pagos como MercadoLibre. A esto se le sumó una serie de perspectivas pedagógicas desde el diseño y la pedagogía de escaneo de información, como las utilizadas en Duolingo o la presencia de material de apoyo y tutoriales bajo el concepto de la experiencia Onboarding [22] para usuarios primerizos o no frecuentes.

\section{Diseño UX}

Superada la etapa de referenciación para la construcción de la plataforma, se prosiguió con el desarrollo de los prototipos de cada módulo y cada una de las funcionalidades requeridas. Es necesario destacar que desde la perspectiva de diseño se buscó mantener una imagen similar de la plataforma desde la vista de administrador tanto como la vista de ciudadano (dos roles diferentes) para facilitar las modificaciones pertinentes y no requerir perfiles profundamente especializados en desarrollo o programación.

A medida que se elaboraron los wireframes y mockups de la plataforma, se aplicaron dos estrategias para tener la experiencia de interacción de usuarios reales y su realimentación en miras de lograr un mejor producto. Para ello, se citaron a 30 personas residentes en Bogotá, con perfiles que se distribuían en cuatro grupos etarios diferentes: 14 a 20 años, 21 a 35 años, 36 a 55 años y adultos mayores. Además, se buscó que las personas participantes en las pruebas tuvieran interés en procesos políticos de la ciudad, es decir, que fueran líderes sociales, activistas políticos o participantes en las diversas convocatorias de estímulos e iniciativas para la ciudad. La mayoría de personas cumplieron este perfil y al menos cinco fueron ciudadanos sin antecedentes de interés político pero interesados en el modelo de presupuestos participativos.

Ocho de las personas convocadas para pruebas de usuario participaron en sesiones de la metodología Card Sorting [23], que permite crear de una manera participativa la arquitectura de los sitios web siguiendo rasgos intuitivos y percepciones lógicas de los usuarios. Los participantes en este taller tenían a su disposición todos los elementos de contenido de los módulos y sugerían cuál debería ser el orden lógico de estos dentro de la plataforma.

Una vez construida la versión inicial de la plataforma, se realizaron sesiones de Experiencia de Usuario [24] para evaluar de forma crítica el desempeño de la plataforma en términos visuales, de interacción y funcionalidad. Allí, los usuarios pudieron hacer sugerencias y denotar inconsistencias en el uso de la plataforma. Las sugerencias fueron atendidas y se realizaron los ajustes pertinentes para incrementar la calidad y robustez del sitio.

\section{Enfoque de Desarrollo de Software}

El trabajo de desarrollo de software arrancó casi de manera paralela a las elaboraciones del equipo de diseño de LAB101. En primera instancia se definieron los requerimientos específicos por parte de la Secretaría de Gobierno para el uso de la plataforma y luego se decidió el lenguaje de programación, las bases de datos y la arquitectura pertinente. Para el desarrollo de la plataforma la Secretaría de Gobierno formuló el requerimiento de alta disponibilidad, es decir que fuera una plataforma con la envergadura para soportar un millón de usuarios diarios y tránsito pesado de información durante el día. Otro requerimiento, que incluso constituyó un reto hasta la entrega del producto, fue la característica de interoperabilidad, esto significa que la plataforma Bogotá Participa y sus tres módulos debían hacer parte de una plataforma con otros servicios y otras funcionalidades creados por la Alcaldía de la ciudad y/o entidades del orden nacional.

La búsqueda del cumplimiento de estos requerimientos incentivó al equipo a crear soluciones desde la arquitectura it en términos de lógica, soporte y manejo de incidencias, comúnmente conocido como el desarrollo de Back End. En términos de lenguaje de programación se hizo uso de Typescript para generar blindaje y un proxy inverso para proporcionar seguridad. En términos de lenguajes, también se incluyeron elementos de Javascript; Nest JS y React JS.

La escogencia del patrón de base de datos se hizo siguiendo la solicitud de la Secretaría de Gobierno de Bogotá de usar una base de datos tipo relacional, ya que permiten correlaciones e intersecciones a partir de elementos comunes 
como la identificación de los ciudadanos y elementos únicos. La base de datos relacional fue gestionada a través de Postgresql.

En términos generales el proceso de desarrollo se resume en los siguientes 4 pasos: 1. Planeación, requerimientos del cliente y negociación. 2. Diseños aprobados. 3. Requerimiento de Base de Datos relacional 3. Desarrollo de Front End (lo visible al usuario y profundamente conectado con las propuestas de diseño gráfico) y Back end (lógica y reglas de negocio de la funcionalidad). 4. Revisiones QA y entrega de Producto.

Adicionalmente, se enfrentaron retos a nivel técnico

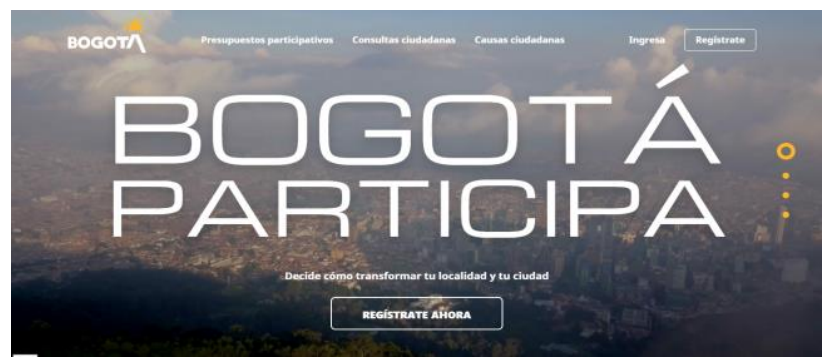

debido a la complejidad y características específicas que se requerían para la construcción de la solución tecnológica, entre ellos el desarrollo Back End que permitiera la funcionalidad de la Billetera Digital. Por un lado, estaba la necesidad de responder a la división administrativa con la que cuenta la ciudad de Bogotá, que permitiera determinar la pertenencia de una persona a alguna de las 20 localidades con las que cuenta actualmente la ciudad. Por otro lado, se requirió un desarrollo robusto en términos de poder garantizar aspectos tales como la privacidad y seguridad de la información que iban a depositar los ciudadanos.

Para poder cumplir con estos requerimientos, lo que se determinó por parte del Distrito fue articular otros sistemas de información que cuentan con protocolos de seguridad y acceso a datos de validación de la cédula de ciudadanía (esto para poder dar cierto nivel de privacidad y confiabilidad de quien ingresaba a la plataforma) y acceso a la plataforma de autenticación creada por la administración distrital, de modo que la plataforma Bogotá Participa solo pudiera acceder a estos datos bajos estándares de uso y no almacenamiento en bases de datos de dicha información.

\section{RESULTADOS}

Home Bogotá Participa

Fig. 1 Plataforma Bogotá Participa disponible en https://participacion.gobiernoabiertobogota.gov.co/

Los resultados concretos de los procesos descritos se traducen en la construcción de la plataforma Bogotá Participa, sus tres módulos y la guía paso a paso Dominó para el uso de la plataforma y la postulación de propuestas por parte de los usuarios. A continuación, se describen en detalle los resultados logrados.

\section{A. Plataforma Bogotá Participa}

La plataforma consta de tres módulos: Presupuestos participativos, Consultas Ciudadanas y Causas Ciudadanas. En el primer módulo los usuarios encontrarán la línea de tiempo con las etapas de desarrollo del modelo de presupuestos participativos desde la postulación de propuestas, pasando por la asignación de dineros hasta la aprobación por parte de la Alcaldía, en este módulo los participantes pueden elegir la localidad de Bogotá en la que residen y postular una propuesta acorde a las necesidades de su comunidad.

En los tres meses que estuvo al aire, recibió una participación de 120.000 usuarios, los cuales definieron la priorización del gasto de hasta el $50 \%$ de los presupuestos asignados a sus localidades. Dichas iniciativas están siendo implementadas por la Alcaldía durante el año 2021. Adicionalmente, en el segundo semestre del año el ejercicio será nuevamente llevado a cabo.

En el segundo módulo, los usuarios podrán revisar las propuestas abiertas, evaluarlas y manifestar su apoyo. En el tercero, se promueve la búsqueda dar afinidad con causas ciudadanas e integrarse con usuarios interesados en las mismas problemáticas. En este sentido, ambos módulos están listos para ser utilizados a disposición de la Alcaldía durante el segundo semestre del 2021.

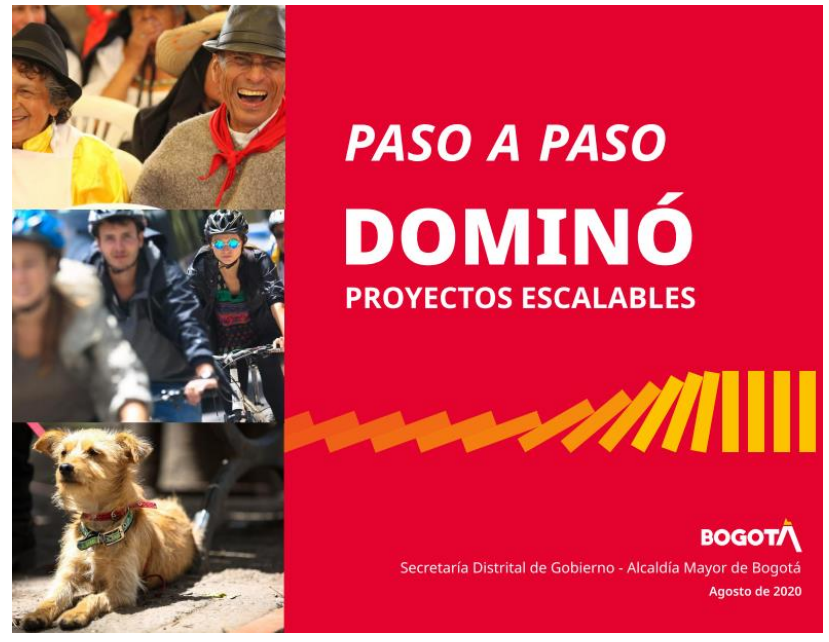

Home Bogotá Participa

Fig. 2 Guía Dominó; Fuente: Elaboración propia LAB101 UNAL.

La guía Dominó buscó ser una herramienta que orientara a los ciudadanos en la creación de propuestas a postular en la 
plataforma Bogotá Participa. El paso a paso fue concebido bajo la mirada interdisciplinaria de profesionales en antropología y publicidad para generar cercanías con la comunidad en general. El nombre Dominó proviene de la doble acepción de la palabra. En primer lugar, el juego Dominó, haciendo alusión a que una propuesta pequeña puede movilizar y desencadenar reacciones que beneficien a la comunidad. El segundo significado posible va conectado con la idea de conocimiento y dominación de un oficio o de una realidad. Se entrelaza estrechamente con el significante de la experticia, y que quienes tienen mayor conocimiento sobre las necesidades locales son los ciudadanos que las vivencian, por lo tanto, las propuestas de solución desde su conocimiento son altamente valiosas.

\section{CONCLUSIONES}

El acceso a espacios participativos y de incidencia democrática se ha convertido en una necesidad no solamente desde el punto de vista de gobernabilidad, sino también como una alternativa que a través de tecnologías de la información y las comunicaciones permite acercar a poblaciones que bajo otras circunstancias y condiciones (como por ejemplo la pandemia ocasionada por el COVID-19) no hubiese permitido darles acceso.

El desarrollo de una herramienta tecnológica como la presentada en este documento, es un reto técnico, pero sobre todo cultural y político, con el fin de lograr enganchar o conectar las visiones que desde la ciudadanía se tienen respecto a la priorización de sus proyectos, frente a las limitaciones y condiciones que desde el sector gubernamental se tienen para la destinación de recursos públicos.

Una recomendación a futuro para este tipo de iniciativas es complementar una estrategia que fomente el uso de estas herramientas tecnológicas en la ciudadanía. Desde el punto de vista técnico, evolucionar las cualidades de la plataforma para visualizar y procesar datos, creando indicadores que posibiliten un seguimiento no solamente a la participación, sino al posterior desarrollo de los proyectos que se prioricen a través de este tipo de mecanismos.

\section{AGRADECIMIENTOS}

A la Secretaría Distrital de Gobierno de la Alcaldía Mayor de Bogotá y particularmente a los funcionarios que interactuaron a lo largo del proyecto por confiar en nuestro laboratorio de innovación UNAL, financiar este proceso de creación tecnológica con impacto social y político, además de construir conjuntamente las diferentes iteraciones a lo largo del proyecto. También especial mención al equipo de trabajo que hizo parte del desarrollo de esta iniciativa. En la página https://participacion.gobiernoabiertobogota.gov.co/acerca de encontrarán más información de las personas que hicieron posible dicho proyecto.

\section{BIBLIOGRAFÍA}

[1] A. R. Corona, «Porto Alegre, un modelo de presupuesto participativo», Rev. Polis México, vol. 1, n. ${ }^{\circ}$ 0, Art. n. ${ }^{\circ}$ 0, 2004.

[2] T. G. Souza Ubiratán de, Presupuesto Participativo. Ediciones Trilce, 1999.

[3] H. R. Gilman, «Engaging Citizens: Participatory Budgeting and the Inclusive Governance Movement within the United States», p. 32, 2016.

[4] B. de S. Santos, Democracia y participación: el ejemplo del presupuesto participativo de Porto Alegre. Editorial Abya Yala, 2004.

[5] Y. Cabannes, «The impact of participatory budgeting on basic services: municipal practices and evidence from the field», Environ. Urban., vol. 27, pp. 257-284, abr. 2015, doi: $10.1177 / 0956247815572297$.

[6] B. de SOUSA SANTOS, «Participatory Budgeting in Porto Alegre: Toward a Redistributive Democracy», Polit. Soc., vol. 26, n. ${ }^{\circ} 4$, pp. 461-510, dic. 1998, doi: 10.1177/0032329298026004003.

[7] B. Wampler, «A Guide to Participatory Budgeting», p. 32.

[8] B. Goldfrank, «LOS PROCESOS DE "PRESUPUESTO PARTICIPATIVO" EN AMÉRICA LATINA: ÉXITO, FRACASO Y CAMBIO», Rev. Cienc. Política Santiago, vol. 26, n. ${ }^{\circ} 2$, pp. 3-28, 2006, doi: 10.4067/S0718-090X2006000200001.

[9] C. A. B. Becerra, Presupuesto participativo: herramienta para la democracia. 2011. Accedido: jun. 25, 2021. [En línea]. Disponible en: https://dialnet.unirioja.es/servlet/libro?codigo $=568238$

[10] R. Annunziata, «El impacto de los Presupuestos Participativos online en el tipo de proyectos: un análisis de la experiencia de BAElige», Rev. CLAD Reforma Democr., n. ${ }^{\circ}$ 77, p. 30, 2020.

[11] F. A. R. Angel y L. T. L. Simonian, «El presupuesto participativo de Pasto: la dinámica participativa y las principales razones de la continuidad», Pap. Político, vol. 24, n. ${ }^{\circ}$ 2, Art. n. ${ }^{\circ}$ 2, 2019, doi: 10.11144/Javeriana.papo24-2.pppd.

[12] J. Cardona Cuervo, J. C. Yepes Ocampo, y A. Guzmán Rendón, «Presupuesto participativo en Armenia, Cartago, Manizales y Pereira, 2014: disputa de ciudadanos y gobierno local», jun. 2017, Accedido: jun. 25, 2021. [En línea]. Disponible en:

https://repository.ucc.edu.co/handle/20.500.12494/1871

[13] «Caja de Herramientas Encuentros Ciudadanos y Presupuestos Participativos», Instituto Distrital de la Participación y Acción Comunal. https://www.participacionbogota.gov.co/caja-deherramientas-encuentros-ciudadanos-y-presupuestos-participativos (accedido jun. 25, 2021).

[14] S. Nadkarni y R. Prügl, «Digital transformation: a review, synthesis and opportunities for future research», Manag. Rev. Q., vol. 71, n. ${ }^{\circ} 2$, pp. 233-341, abr. 2021, doi: 10.1007/s11301-020-00185-7.

[15] J. S. Gans, «Keep Calm and Manage Disruption», MIT Sloan Management Review. https://sloanreview.mit.edu/article/keep-calmand-manage-disruption/ (accedido jun. 30, 2021).

[16] «Inicio». https://www.lab101.co/ (accedido jun. 30, 2021).

[17] K. Hansson, K. Belkacem, y L. Ekenberg, «Open Government and Democracy: A Research Review», Soc. Sci. Comput. Rev., vol. 33, n. ${ }^{\circ}$ 5, pp. 540-555, oct. 2015, doi: 10.1177/0894439314560847.

[18] J. Bates, «The strategic importance of information policy for the contemporary neoliberal state: the case of Open Government Data in the United Kingdom», Gov. Inf. Q., vol. 31, n. ${ }^{\circ}$ 3, Art. n. ${ }^{\circ}$ 3, 2014.

[19] M. Mahalakshmi y D. M. Sundararajan, Traditional SDLC Vs Scrum Methodology - A Comparative Study.

[20] S. Goldsmith, Universal Design. London: Routledge, 2000. doi: $10.4324 / 9780080520209$.

[21] C. D. Spradling, «SPEC CPU2006 benchmark tools», ACM SIGARCH Comput. Archit. News, vol. 35 , n. ${ }^{\circ} 1$, pp. 130-134, mar. 2007, doi: 10.1145/1241601.1241625.

[22] A. Ju, H. Sajnani, S. Kelly, y K. Herzig, «A Case Study of Onboarding in Software Teams: Tasks and Strategies», en 2021 IEEE/ACM 43rd International Conference on Software Engineering 
(ICSE), may 2021, pp. 613-623. doi

10.1109/ICSE43902.2021.00063.

[23] S. Hannah, «Sorting Out Card Sorting: Comparing Methods for Information Architects, Usability Specialists, and Other

Practitioners», nov. 2008, Accedido: jun. 25, 2021. [En línea]. Disponible en:

https://scholarsbank.uoregon.edu/xmlui/handle/1794/7818

[24] Y. H. Montero, «Experiencia de Usuario: Principios y Métodos», p. 139. 